\title{
Time-Delayed Feedback Control of a Hydraulic Model Governed by a Diffusive Wave System
}

\author{
Boumediène Chentouf $(\mathbb{D}$ and Nejib Smaoui \\ Kuwait University, Faculty of Science, Department of Mathematics, Safat 13060, Kuwait \\ Correspondence should be addressed to Boumediène Chentouf; chenboum@hotmail.com
}

Received 11 September 2019; Accepted 30 December 2019; Published 9 March 2020

Academic Editor: Guang Li

Copyright (C) 2020 Boumediène Chentouf and Nejib Smaoui. This is an open access article distributed under the Creative Commons Attribution License, which permits unrestricted use, distribution, and reproduction in any medium, provided the original work is properly cited.

\begin{abstract}
This paper is concerned with the feedback flow control of an open-channel hydraulic system modeled by a diffusive wave equation with delay. Firstly, we put forward a feedback flow control subject to the action of a constant time delay. Thereafter, we invoke semigroup theory to substantiate that the closed-loop system has a unique solution in an energy space. Subsequently, we deal with the eigenvalue problem of the system. More importantly, exponential decay of solutions of the closed-loop system is derived provided that the feedback gain of the control is bounded. Finally, the theoretical findings are validated via a set of numerical results.
\end{abstract}

\section{Introduction}

The dynamics of irrigation canals are often described by nonlinear complex partial differential equations derived from the conservation of mass and momentum. Among several models available in the literature, the de SaintVenant system $[1,2]$ has been standing at the forefront of the modeling and analysis of irrigation canals for decades. The system consists of two nonlinear coupled hyperbolic partial differential equations:

$$
\begin{gathered}
\frac{\partial Z}{\partial t}+\frac{\partial q}{\partial x}=L_{d} \\
\frac{\partial q}{\partial t}+\frac{\partial}{\partial x}\left(\frac{q^{2}}{Z}\right)+g Z \frac{\partial E}{\partial x}=-g Z S+r V L_{d}
\end{gathered}
$$

where $x$ is the spatial location (m) and $t$ is the time (s). Furthermore, $E$ represents the absolute water surface elevation $(\mathrm{m}), Z(x, E)$ is the wetted cross-sectional area $\left(\mathrm{m}^{2}\right), q(x, t)$ is the flow discharge $\left(\mathrm{m}^{3} / \mathrm{s}\right), S(q, E, x)$ the friction slope, and $L_{d}(x, t)$ is the lateral discharge $\left(\mathrm{m}^{2} / \mathrm{s}\right)$, which can be interpreted as an inflow if $L_{d}>0$ and an outflow when $L_{d}<0$. Moreover, $r=0$ as long as $L_{d}>0$, whereas if $L_{d}<0$, then $r=1$. Finally, $V(x, t)$ is the mean velocity $(\mathrm{m} / \mathrm{s})$ in section $Z$ and $g$ the gravitational acceleration $\left(\mathrm{m}^{2} / \mathrm{s}\right)$.

As mentioned above, the conservation of mass is reflected by equation (1) and the conservation of momentum is translated by (2) (see also [3] for the 2D model).

It turned out that, in practice, system (1)-(2) cannot be well studied unless the geometry of the irrigation canal is completely known and also the roughness coefficient. As pointed out in $[2,4]$, such requirements may not be reachable for long rivers. This has motivated many authors to derive other models. For instance, the following model has been used in $[4,5]$ :

$$
\frac{\partial q}{\partial t}(x, t)=\alpha \frac{\partial^{2} q}{\partial x^{2}}(x, t)-\beta \frac{\partial q}{\partial x}(x, t),
$$

where the positive constants $\alpha$ and $\beta$ are the diffusion and the celerity, respectively. Specifically, the above equation has been derived under several assumptions such as assuming that the lateral inflow is minimum, the flow variations as well as the bed slope of the long river are small, and the inertia terms $(\partial q / \partial t)+(\partial / \partial x)\left(q^{2} / Z\right)$ are negligible with respect to $g Z(\partial E / \partial x)$ (for further discussion on the model, the reader is referred to $[4,6])$. However, for sake of clarity, we shall 
give some details about the derivation of (3). In fact, under the above assumptions, system (1)-(2) have the following form:

$$
\begin{gathered}
\frac{\partial q}{\partial x}=-W \frac{\partial E}{\partial t} \\
S=-\frac{\partial E}{\partial x}
\end{gathered}
$$

where $W$ is the water surface width. Differentiating (4) and (5), it follows that

$$
W^{2} \frac{\partial S}{\partial t}=W \frac{\partial^{2} q}{\partial x^{2}}-\frac{\partial W}{\partial x} \frac{\partial q}{\partial x}
$$

On the other hand, we have

$$
\begin{gathered}
\left(\frac{\partial W}{\partial x}\right)_{t}=\left(\frac{\partial W}{\partial E}\right)_{x} \frac{\partial E}{\partial x}+\left(\frac{\partial W}{\partial x}\right)_{E}=-S\left(\frac{\partial W}{\partial E}\right)_{x}+\left(\frac{\partial W}{\partial x}\right)_{E} \\
\left(\frac{\partial S}{\partial t}\right)=\frac{\partial S}{\partial q}\left(\frac{\partial q}{\partial t}\right)_{x}+\frac{\partial S}{\partial E}\left(\frac{\partial E}{\partial t}\right)_{x}=\frac{\partial S}{\partial q}\left(\frac{\partial q}{\partial t}\right)_{x}-\frac{1}{W} \frac{\partial S}{\partial E} \frac{\partial q}{\partial x}
\end{gathered}
$$

where $((\partial f / \partial u)(u, v))_{v}$ denotes the variation of $f$ with respect to $u$ when $v$ is fixed. Combining these identities with (6) gives

$$
\frac{\partial q}{\partial t}=\alpha(q, E, x) \frac{\partial^{2} q}{\partial x^{2}}-\beta(q, E, x) \frac{\partial q}{\partial x}
$$

where

$$
\begin{aligned}
\alpha(q, E, x) & =\frac{1}{W(\partial S / \partial q)} \\
\beta(q, E, x) & =\frac{1}{W(\partial S / \partial q)}\left(\frac{\partial S}{\partial E}-\frac{1}{W}\left[\frac{\partial W}{\partial E}(-S)+\frac{\partial W}{\partial x}\right]\right) \\
& =\frac{1}{W^{2}(\partial S / \partial q)}\left(\frac{\partial(W S)}{\partial E}-\frac{\partial W}{\partial x}\right) .
\end{aligned}
$$

Finally, it suffices to linearize the latter around a reference flow and come up with the diffusive wave (3).

It is worth mentioning that the model under consideration (3) is not only of practical interest but also does not require too many physical parameters of the canal. Indeed, only two parameters are required in order to characterize the water flow, namely, celerity and diffusivity.

The control and stabilization problem of (3) has been addressed in several research papers by adopting different approaches and strategies. To name few, using discretization techniques such as the finite difference method or using a finite dimensional approximation in the frequency domain, the authors provided exhaustive studies, where the main concern was controlling (3) and/or estimating its parameters [1, 4-20] (see also [21] for a nonlinear case). Later, using the tools of control theory of partial differential equations [22], the authors in [23-25] tackled the problem of robust regulation of (3) by proposing a proportional and/or integral controller. In a recent article [6], the authors dealt with the output feedback stabilization of model (3). To be more precise, the following system has been put forward in [6]:

$$
\frac{\partial q}{\partial t}(x, t)=\alpha \frac{\partial^{2} q}{\partial x^{2}}(x, t)-\beta \frac{\partial q}{\partial x}(x, t)+b(x) \mathcal{U}(t)
$$

together with the boundary conditions and the output observation

$$
\left\{\begin{array}{l}
q(0, t)=\frac{\partial q}{\partial x}(\ell, t)=0, \\
y(t)=q(\ell, t)
\end{array}\right.
$$

where $\mathcal{U}(t)$ is the control, $y(t)$ is the output, and $b(x)$ is the spatial distribution of the actuator. Then, it has been shown that the closed-loop system is exponentially stable under the action of the control $\mathcal{U}(t)=\kappa q(\ell, t)$, where $\kappa$ is the feedback gain.

Up to our knowledge, the stabilization problem of (11) with delay has not been examined in the literature. In fact, as previously mentioned, the time delay has not been taken into account in [6], where the exponential stability result is obtained under the condition $|\kappa|<(2 \sqrt{\alpha \beta} /(\|b\| \ell))$. In this article, we go a step further in the sense that we do take into consideration the presence of the time delay in the input control. This is motivated by the fact that a timedelay naturally occurs in practice due to the time factor needed for the communication among the controllers, the sensors, and the actuators of systems. Obviously, when a controller monitors the state of a system, it will make an adjustment (if a need arises) based on its observation. Notwithstanding, such an adjustment surely requires some time to be completed as it can never be conducted in an instantaneous manner. It is also of great interest to point out that the occurrence of a delay in a system could cause a poor performance of the control and could sometime generate instabilities in the system [26-28]. Based on the above discussion, the study of asymptotic behavior of the solutions of system (11) and (12) with input delay is of theoretical as well as practical prominence. The reader can also consult [26-35], where the effect of time delays in several types of partial differential equations (PDEs) has been studied.

The main contribution of this paper is twofold:

(1) Extend the mathematical findings of $[6,36]$ where the presence of time delay has not been considered in the feedback law. In fact, our control is

$$
\mathcal{U}(t)=\kappa q(\ell, t-\tau),
$$

where $\kappa>0$ is the feedback gain and $\tau>0$ is the delay. Then, it is shown that the closed-loop system (11)-(13) is stable even though a delay supervenes in the proposed control. This result is obtained at the expense of a reasonable restriction on the feedback gain, which has been also used in [6] even if there was no delay in the control. 
(2) An extensive numerical study is conducted in order to validate our results. More precisely, adopting the Chebyshev collocation that uses the backward Euler method and the Gauss-Lobatto points, for the delayed system (11)-(13), we are able to illustrate, for different values of the delay and the physical parameters of the canal, the stability result.

The rest of this paper is organized as follows. In Section 2, the closed-loop system is formulated in an evolution equation and preliminary results are put forward for the open-loop system. Next, we prove in Section 3 that our problem is well posed in the sense of semigroup theory of linear operators. In Section 4, we characterize the spectrum of the closed-loop system. Section 5 is devoted to the proof of the main result of this work, namely, the exponential stability of the closed-loop system. In Section 6, we show the relevance of the theoretical results through several numerical simulations. Finally, our conclusions are given in Section 7.

\section{Problem Set-Up and Preliminaries}

In this section, the closed-loop system is formulated as a differential equation in an appropriate functional space. To do so, the notations used in the paper are presented, as well as some definitions and theorems. Finally, a comprehensive study of the open-loop system is provided.

Given two initial data $q_{0}$ and $u_{0}$, the closed-loop system (11)-(13) can be written as follows:

$$
\begin{cases}\frac{\partial q}{\partial t}(x, t)=\alpha \frac{\partial^{2} q}{\partial x^{2}}(x, t)-\beta \frac{\partial q}{\partial x}(x, t)+\kappa b(x) q(\ell, t-\tau), & (x, t) \in(0, \ell) \times(0, \infty), \\ q(0, t)=\frac{\partial q}{\partial x}(\ell, t)=0, & t>0, \\ q(x, 0)=q_{0}(x), & x \in(0, \ell), \\ q(\ell, \theta)=u_{0}(\theta), & \theta \in(-\tau, 0) .\end{cases}
$$

For sake of clarity, we recall that

$$
L^{2}(0, \ell)=\left\{\varphi \text { is measurable and } \int_{0}^{\ell} \varphi^{2}(x) d x<\infty\right\},
$$

is endowed with its standard norm $\|\varphi\|=\left(\int_{0}^{\ell} \varphi^{2}(x) d x\right)^{1 / 2}$. Furthermore, the Sobolev space

$$
H^{n}(0, \ell)=\left\{\varphi:(0, \ell) \longrightarrow \mathbb{R} ; \varphi^{(n)} \in L^{2}(0, \ell), \quad \text { for } n=0,1,2, \ldots\right\},
$$

is equipped with the usual norm $\|\varphi\|_{H^{n}(0, \ell)}=\sum_{i=0}^{n}\left\|\varphi^{(i)}\right\|_{L^{2}(0, \ell)}$. In view of the well-known change of state variable $[33,37]$,

$$
u(\rho, t)=q(\ell, t-\tau \rho), \quad \rho \in(0,1),
$$

the closed-loop system (14) becomes

$$
\begin{cases}\frac{\partial q}{\partial t}(x, t)=\alpha \frac{\partial^{2} q}{\partial x^{2}}(x, t)-\beta \frac{\partial q}{\partial x}(x, t)+\kappa b(x) u(1, t), & (x, t) \in(0, \ell) \times(0, \infty), \\ \tau \frac{\partial u}{\partial t}(\rho, t)+\frac{\partial u}{\partial \rho}(\rho, t)=0, & (\rho, t) \in(0,1) \times(0, \infty), \\ q(0, t)=\frac{\partial q}{\partial x}(\ell, t)=0, & t>0, \\ q(x, 0)=q_{0}(x), & x \in(0, \ell), \\ u(\rho, 0)=u_{0}(-\tau \rho), & \rho \in(0,1),\end{cases}
$$


whereupon consider the state space

$$
\mathscr{H}=L^{2}(0, \ell) \times L^{2}(0,1),
$$

equipped with the following inner product:

$$
\langle(q, u),(\widetilde{q}, \widetilde{u})\rangle_{\mathscr{H}}=\int_{0}^{\ell} q \widetilde{q} d x+\tau \beta \int_{0}^{1} u \widetilde{u} d x .
$$

Obviously, the norm induced by such an inner product is equivalent to the usual norm of the space $L^{2}(0, \ell) \times$ $L^{2}(0,1)$. For convenience, we shall often denote by $f_{v}$ the derivative of $f$ with respect to the variable $v$. Then, let us assume that the spatial actuator $b(\cdot) \in L^{2}(0, \ell)$ (one may suppose that $\left.b(\cdot) \in L^{\infty}(0, \ell)\right)$ and consider the system operator:

$$
\begin{gathered}
\mathscr{D}(\mathscr{A}):=\left\{(q, u) \in H^{2}(0, \ell) \times H^{1}(0, \ell) ;\right. \\
\left.q(0)=q_{x}(\ell)=0, \text { and } u(0)=q(\ell)\right\}, \\
\mathscr{A}(q, u):=\left(\alpha q_{x x}-\beta q_{x}+\kappa b(\cdot) u(1),-\frac{u_{\rho}}{\tau}\right) .
\end{gathered}
$$
form:

Thereby, system (18) can be brought to the following

$$
\left\{\begin{array}{l}
\dot{\phi}(t)=\mathscr{A} \phi(t) . \\
\phi(0)=\phi_{0},
\end{array}\right.
$$

where $\phi=(q, u)$ and $\phi_{0}=\left(q_{0}, u_{0}\right)$.

2.1. Open-Loop System. First and foremost, we shall investigate the main properties of the uncontrolled system which is given by

$$
\left\{\begin{array}{l}
\frac{\partial R(x, t)}{\partial t}=\alpha \frac{\partial^{2} R(x, t)}{\partial x^{2}}-\beta \frac{\partial R(x, t)}{\partial x}, \\
R(0, t)=R_{x}(\ell, t)=0 .
\end{array}\right.
$$

Taking the Hilbert state space $L^{2}(0, \ell)$ equipped with the usual inner product, system (24) can be written in the abstract form $\dot{R}(t)=\mathscr{A}_{0} R(t)$, where $\mathscr{A}_{0}$ is an unbounded linear operator defined by

$$
\begin{aligned}
\mathscr{D}\left(\mathscr{A}_{0}\right) & =\left\{R \in H^{2}(0, \ell) ; R(0)=R_{x}(\ell)=0\right\}, \\
\mathscr{A}_{0} R & =\alpha R_{x x}-\beta R_{x} .
\end{aligned}
$$

The following results will be systematically used.

Theorem 1 (Lumer-Phillips) (see [38]). Given a linear operator $\mathscr{P}$ on a Hilbert space $\mathscr{Y}$ with $\overline{D(\mathscr{P})}=\mathscr{Y}$, if $\mathscr{P}$ is dissipative and there is a scalar $\lambda_{0}$ such that $\lambda_{0} I-\mathscr{P}$ is onto $\mathscr{Y}$, then $\mathscr{P}$ generates a $C_{0}$ semigroup of contractions on $\mathcal{Y}$.

Theorem 2 (see [39]). Assume that $\mathscr{P}$ be a closed densely defined operator on a Hilbert space $y$ satisfying the following property: there exists $\eta \in \mathbb{R}$ such that

$$
\begin{gathered}
\langle\mathscr{P} v, v\rangle_{\mathscr{Y}} \leq \eta\|v\|_{\mathscr{Y}}^{2}, \quad \forall v \in \mathscr{D}(\mathscr{P}), \\
\left\langle\mathscr{P}^{*} v, v\right\rangle_{\mathscr{Y}} \leq \eta\|v\|_{\mathscr{Y}}^{2}, \quad \forall v \in \mathscr{D}\left(\mathscr{P}^{*}\right),
\end{gathered}
$$

where $\mathscr{P}^{*}$ is the adjoint operator of $\mathscr{P}$. Then, the operator $\mathscr{P}$ generates a $C_{0}$-semigroup $S(t)$ satisfying $\|S(t)\|_{\mathscr{L}(\mathscr{Y})} \leq e^{\eta t}$.

The basic properties of the operator $\mathscr{A}_{0}$ (see (25)) are summarized below.

Proposition 1. The operator $\mathscr{A}_{0}$ defined by (25) generates a $C_{0}$-semigroup of contractions $S_{0}(t)$ on $L^{2}(0, \ell)$. In addition, the resolvent of $\mathscr{A}_{0}$ is compact and the spectrum $\sigma\left(\mathscr{A}_{0}\right)$ consists entirely of isolated eigenvalues with finite multiplicities.

Proof. Using (25) and integrating by parts, we obtain

$$
\left\langle\mathscr{A}_{0} R, R\right\rangle_{L^{2}(0, \ell)}=-\alpha \int_{0}^{\ell} R_{x}^{2}(x) d x-\frac{\beta}{2} R^{2}(\ell) \leq 0,
$$

for any $R \in \mathscr{D}\left(\mathscr{A}_{0}\right)$. Thereby, $\mathscr{A}_{0}$ is dissipative.

Given now $Q \in L^{2}(0, \ell)$, a direct calculation yields

$$
\begin{aligned}
R(x):= & \mathscr{A}_{0}^{-1} Q=\frac{\alpha}{\beta}\left[\int_{0}^{x}\left(e^{(\alpha / \beta)(x-s)}-1\right) Q(s) d s\right. \\
& \left.+\left(1-e^{(\alpha / \beta) x}\right) \int_{0}^{\ell} e^{-(\alpha / \beta) x} Q(s) d s\right] .
\end{aligned}
$$

Therefore, the resolvent equation $\mathscr{A}_{0} R=Q$ has a unique solution $R \in \mathscr{D}\left(\mathscr{A}_{0}\right)$ for each $Q \in L^{2}(0, \ell)$. Evoking Theorem 1 , one can deduce that $\mathscr{A}_{0}$ generates a $C_{0}$-semigroup of contractions $S_{0}(t)$ on $L^{2}(0, \ell)$. On the other hand, using the Sobolev embedding theorem [40], we conclude that the resolvent operator of $\mathscr{A}_{0}$ is compact on $L^{2}(0, \ell)$ and hence the spectrum of $\mathscr{A}_{0}$ only has isolated eigenvalues with finite multiplicities [41].

For sake of completeness, let us recall the following definition and theorem.

\section{Definition 1}

(i) A semigroup $S(t)$ is stable on a Hilbert space $\mathscr{Y}$ if $\lim _{t \rightarrow \infty}\|S(t) v\|=0$, for any $v \in \mathcal{Y}$.

(ii) If there exist positive constants $C$ and $\omega$ such that $\|S(t)\| \leq C e^{-\omega t}$, for all $t \geq 0$, then the semigroup is exponentially stable.

Theorem 3 (see [42]). Let $S(t)$ be a $C_{0}$-semigroup in $\mathcal{Y}$ whose generator $\mathscr{P}$ has a compact resolvent. Then, $S(t)$ is stable if and only if it is uniformly bounded and the eigenvalues of $\mathscr{P}$ belong to the left halfplane.

The stability result for the operator $\mathscr{A}_{0}$ (see (25)) is as follows.

Proposition 2. The semigroup $S_{0}(t)$ generated by the operator $\mathscr{A}_{0}$ defined by $(25)$ is exponentially stable in $L^{2}(0, \ell)$. 
Proof. Let $R(x, t)$ be the unique solution stemmed from an initial condition $R_{0}$ in $\mathscr{D}\left(\mathscr{A}_{0}\right)$. Integrating by parts and using the boundary conditions in (25), we have

$$
\frac{1}{2} \frac{d}{d t}\left(\|R(x, t)\|^{2}\right)=-\alpha \int_{0}^{\ell} R_{x}(x, t)^{2}(x) d x-\frac{\beta}{2} R^{2}(\ell, t) .
$$

Moreover, for each $\varphi \in\left\{f \in H^{1}(0, \ell) ; f(0)=0\right\}$, we have the following Wirtinger's inequality [43]:

$$
\int_{0}^{\ell} \varphi^{2}(x, t) d x \leq \frac{4 \ell^{2}}{\pi^{2}} \int_{0}^{\ell} \varphi_{x}^{2}(x, t) d x,
$$

which together with the last estimate imply that $(d / d t)$ $\left(\|R(x, t)\|^{2}\right) \leq-\left(\alpha \pi^{2} /\left(2 \ell^{2}\right)\right)\|R(x, t)\|^{2}$. Solving the above differential inequality, we get $\|R(x, t)\| \leq e^{-(\pi \sqrt{2 \alpha} /(2 \ell)) t}\left\|R_{0}\right\|$, which leads to the exponential stability of $R(x, t)$ in $L^{2}(0, \ell)$, thanks to a classical density argument.

Now, we turn to the characterization of the spectrum of $\mathscr{A}_{0}$.

Proposition 3. The eigenvalues of $\mathscr{A}_{0}$ form the following set of negative real numbers:

$$
\left\{\mu_{n}=-\left(\frac{\ell^{2} \beta^{2}+4 \alpha^{2} e_{n}^{2}}{4 \alpha \ell^{2}}\right) ; n \in \mathbb{N}\right\},
$$

where $e_{n}$ is a nonzero real solution of

$$
2 \alpha e_{n} \cos e_{n}=-\beta \ell \sin e_{n} .
$$

Furthermore, we have $\sup \left\{\mu_{n} ; \mu_{n}\right.$ is an eigenvalue of $\left.\mathscr{A}_{0}\right\}=-\left(\left(\ell^{2} \beta^{2}+4 \alpha^{2} e^{2}\right) /\left(4 \alpha \ell^{2}\right)\right)$, where $e$ is the smallest positive solution of (33).
Proof. It follows from (25) that a complex $\mu$ is an eigenvalue of $\mathscr{A}_{0}$ if and only if the system

$$
\left\{\begin{array}{l}
\alpha R_{x x}-\beta R_{x}-\mu R=0, \\
R(0)=R_{x}(\ell)=0,
\end{array}\right.
$$

has a nontrivial solution. For sake of clarity, let

$$
G_{\mu}=4 \alpha \mu+\beta^{2}, \sigma_{1}=\frac{\beta-\sqrt{G_{\mu}}}{2 \alpha}, \sigma_{2}=\frac{\beta+\sqrt{G_{\mu}}}{2 \alpha} .
$$

Two cases will be treated, namely, $G_{\mu}=0$ and $G_{\mu} \neq 0$.

i) $G_{\mu}=0$, which means that $\mu=-\beta^{2} /(4 \alpha)$ and hence $\sigma_{1}=\sigma_{2}=\beta /(2 \alpha)>0$. Thereby, it is easy to check that (34) has only the trivial solution and hence $\mu=-\beta^{2} /(4 \alpha)$ cannot be an eigenvalue of $\mathscr{A}_{0}$.

ii) $G_{\mu} \neq 0$, i.e., $\sigma_{1} \neq \sigma_{2}$. In such a case, the general solution of (34) is $R(x)=k_{1} e^{\sigma_{1} x}+k_{2} e^{\sigma_{2} x}$, where the constants satisfy the linear system:

$$
\left\{\begin{array}{l}
k_{1}+k_{2}=0 \\
\sigma_{1} e^{\sigma_{1} \ell} k_{1}+\sigma_{2} e^{\sigma_{2} \ell} k_{2}=0
\end{array}\right.
$$

whereupon $\mu$ is an eigenvalue if and only if $\sigma_{1} e^{\sigma_{1} \ell}=\sigma_{2} e^{\sigma_{2} \ell}$, which can be written as follows:

$$
\beta \ell \sinh \zeta+2 \alpha \zeta \cosh \zeta=0,
$$

where $\zeta=\left(\sqrt{G_{\mu}} /(2 \alpha)\right) \ell$. The immediate task is to characterize the solutions of (37). To do so, write $\zeta=a+i e$, where $a$ and $e$ are two real numbers, and using (37) yields

$$
\left\{\begin{array}{l}
e^{-a}[(2 \alpha a-\beta e) \cos e+2 \alpha e \sin e]+e^{a}[(2 \alpha a+\beta e) \cos e-2 \alpha e \sin e]=0 \\
e^{-a}[(\beta e-2 \alpha a) \sin e+2 \alpha e \cos e]+e^{a}[(2 \alpha a+\beta \ell) \sin e+2 \alpha e \cos e]=0
\end{array}\right.
$$

This implies that $a$ and $e$ must obey the following condition:

$$
e^{4 a}\left[(2 \alpha a+\beta \ell)^{2}+4 \alpha^{2} e^{2}\right]=(2 \alpha a-\beta \ell)^{2}+4 \alpha^{2} e^{2} .
$$

It is a simple task to check that the above equation is valid only in the event that $a=0$ and thus (38) becomes $\beta \ell \sin e+2 \alpha e \cos e=0$. Recalling that $\zeta=i e=\left(\sqrt{G_{\mu}} /(2 \alpha)\right) \ell$ and using (35), we obtain the desired result.

The asymptotic expansion of the eigenvalues of $\mathscr{A}_{0}$ is provided in the following proposition.

Proposition 4. Let $\mu_{n}$ be an eigenvalue of system (24). Then, for $n$ large enough, we have

$$
\mu_{n}=-\beta\left(\frac{1}{\ell}+\frac{\beta}{4 \alpha}\right)-\frac{\pi^{2}}{\alpha \ell^{2}}\left(\frac{2 n+1}{2}\right)^{2}+\mathcal{O}\left(\frac{1}{n}\right)+i \mathcal{O}\left(\frac{1}{n}\right) .
$$

Proof. In view of Proposition 3, we know that $\mu$ is an eigenvalue of $\mathscr{A}_{0}$ if and only if $\zeta=\left(\sqrt{G_{\mu}} /(2 \alpha)\right) \ell$ is a solution of (37). In turn, the solution set of (37) admits the two bisectors of the complex plane as symmetrical axes and the origin as a symmetrical center. Thereby, we just need to find the solutions of (37) in a sector $\mathcal{S}$ defined by $\arg (\theta)=v$ with $\epsilon<v<(\pi / 2)+\epsilon$ for $\epsilon$ small. Next, applying Rouché theorem in $\mathcal{S}$, the solutions of (37) have the following asymptotic representation:

$$
\zeta_{n}=\mathcal{O}\left(\frac{1}{n^{2}}\right)+i\left[\frac{\beta L}{2 \alpha \pi n}+\pi\left(\frac{2 n+1}{2}\right)+\mathcal{O}\left(\frac{1}{n^{2}}\right)\right]
$$

for $n$ large enough. Finally, by virtue of (35) and the fact that $\zeta=\left(\sqrt{G_{\mu}} /(2 \alpha)\right) \ell$, it follows that $\mu_{n}=-(\beta / \ell)-\left(\beta^{2} /(4 \alpha)\right)-$ $\left(\pi^{2} /\left(\alpha \ell^{2}\right)\right)(n+(1 / 2))^{2}+\mathcal{O}(1 / n)+i \mathcal{O}(1 / n)$, which is the expression in our proposition. 


\section{Semigroup Generation for the Closed- Loop System}

The main objective of this section is to show that the closedloop system (23) is well posed in the state space $\mathscr{H}=L^{2}(0, \ell) \times L^{2}(0,1)$. The proof of such a desirable result is based on the application of semigroup theory of linear operators. Specifically, we shall use Theorem 2. We have indeed the following result.

Theorem 4. The operator $\mathscr{A}$, defined by (21) and (22), generates a $C_{0}$-semigroup $S(t)$ on the Hilbert space $\mathscr{H}$.

Proof. Let $\phi=(q, u) \in \mathscr{D}(\mathscr{A})$. Invoking (21) and (22) and integrating by parts, we get

$$
\begin{aligned}
\langle\mathscr{A} \phi, \phi\rangle_{\mathscr{H}}= & -\alpha \int_{0}^{\ell} q_{x}^{2}(x) d x-\frac{\beta}{2} q^{2}(\ell)+\kappa u(1) \int_{0}^{\ell} b(x) q(x) d x \\
& -\frac{\beta}{2}\left(u^{2}(1)-u^{2}(0)\right) \\
= & -\alpha \int_{0}^{\ell} q_{x}^{2}(x) d x+\kappa u(1) \int_{0}^{\ell} b(x) q(x) d x-\frac{\beta}{2} u^{2}(1) .
\end{aligned}
$$

Applying Cauchy-Schwartz and Young's inequalities, the latter becomes

$$
\begin{aligned}
\langle\mathscr{A} \phi, \phi\rangle_{\mathscr{H}} \leq & -\alpha \int_{0}^{\ell} q_{x}^{2}(x) d x+\frac{|\kappa| C-\beta}{2} u^{2}(1) \\
& +\frac{|\kappa|\|b\|^{2}}{2 C} \int_{0}^{\ell} q^{2}(x) d x
\end{aligned}
$$

for any positive constant $C$. Choosing $C=\beta /|\kappa|$, we deduce that

$$
\operatorname{Re}\langle\mathscr{A} \phi, \phi\rangle_{\mathscr{H}} \leq \frac{|\kappa|^{2}}{2 \beta}\|b\|^{2} \int_{0}^{\ell} q^{2}(x) d x .
$$

The task ahead is to show that the above inequality also holds for the adjoint operator of $\mathscr{A}^{*}$. First, one can readily verify that the operator $\mathscr{A}$ is closed and its domain is dense in $\mathscr{H}$. Furthermore, the adjoint operator of $\mathscr{A}^{*}$ is given by

$$
\begin{aligned}
& \mathscr{D}\left(\mathscr{A}^{*}\right):=\left\{(f, g) \in H^{2}(0, \ell) \times H^{1}(0,1) ; f(0)=0,\right. \\
& \left.\beta g(0)=\alpha f_{x}(\ell)+\beta f(\ell), \beta g(1)=\kappa \int_{0}^{\ell} b(x) f(x) d x\right\} \\
& \mathscr{A}^{*}(f, g):=\left(\alpha f_{x x}+\beta f_{x},-\frac{g_{\rho}}{\tau}\right), \quad \forall q \in \mathscr{D}\left(\mathscr{A}^{*}\right) .
\end{aligned}
$$

Subsequently, let us consider $(q, u) \in \mathscr{D}\left(\mathscr{A}^{*}\right)$. Integrating by parts twice and using (45) and (46), it follows that

$$
\begin{aligned}
\left\langle\mathscr{A}^{*}(q, u),(q, u)\right\rangle_{\mathscr{\ell}} & =\alpha q_{x}(\ell) q(\ell)-\alpha \int_{0}^{\ell} q_{x}^{2}(x) d x+\frac{\beta}{2} q^{2}(\ell)+\frac{\beta}{2}\left(u^{2}(1)-u^{2}(0)\right) \\
& \leq-\alpha \int_{0}^{\ell} q_{x}^{2}(x) d x-\frac{\alpha^{2}}{2 \beta} q_{x}^{2}(\ell)+\frac{|\kappa|^{2}}{2 \beta}\|b\|^{2} \int_{0}^{\ell} q^{2}(x) d x \\
& \leq \frac{|\kappa|^{2}}{2 \beta}\|b\|^{2} \int_{0}^{\ell} q^{2}(x) d x
\end{aligned}
$$

By virtue of (44) and (47), we conclude, thanks to Theorem 2, that the operator $\mathscr{D}(\mathscr{A})$ generates a $C_{0}$-semigroup $S(t)$ satisfying $\|S(t)\|_{\mathscr{L}(\mathscr{H})} \leq \exp \left(\left(|\kappa|^{2}\|b\|^{2} /\right.\right.$ $(2 \beta)) t)$.

Remark 1. A direct consequence of Theorem 4 is that the $C_{0}$-semigroup $S(t)$ of the closed-loop system is not necessarily uniformly bounded for all values of the feedback gain $\kappa>0$. One can explain this property by recalling that the generator $\mathscr{A}$ of $S(t)$ is not dissipative for all values of the feedback gain $\kappa>0$, unlike the uncontrolled system whose operator $\mathscr{A}_{0}$ is dissipative and hence its semigroup is contractive (see Proposition 1).

\section{Eigenvalue Problem}

It is the intention of this section to describe the spectrum and the resolvent set of the closed-loop system. To this end, we first prove that the system operator $\mathscr{A}$ is indeed a discrete operator.

Theorem 5. The operator $(\lambda I-\mathscr{A})^{-1}$ is compact on $\mathscr{H}$ for some $\lambda>0$. Therewith, $\mathscr{A}$ is a discrete operator and the spectrum set $\sigma(\mathscr{A})$ consists of isolated eigenvalues with finite multiplicities. Moreover, if $\lambda \in \rho(\mathscr{A})$, then the resolvent operator $(\lambda I-\mathscr{A})^{-1}$ is compact.

Proof. For arbitrary $(f, g) \in \mathscr{H}$, let us solve the resolvent equation with $\lambda>0$ :

$$
\left\{\begin{array}{l}
\alpha q_{x x}(x)-\beta q_{x}(x)-\lambda q(x)+\kappa b(x) u(1)=-f(x), \\
u_{\rho}(\rho)+\lambda \tau u(\rho)=\tau g(\rho), \\
u(0)=q(\ell), q(0)=q_{x}(\ell)=0 .
\end{array}\right.
$$

Solving the equation of $u$ in the above system and using the condition $u(0)=q(\ell)$, we obtain 


$$
u(\rho)=q(\ell) e^{-\lambda \tau \rho}+\tau \int_{0}^{\rho} g(r) e^{-\lambda \tau(\rho-r)} d r
$$

and hence

$$
\varpi:=u(1)=q(\ell) e^{-\lambda \tau}+\tau \int_{0}^{1} g(r) e^{-\lambda \tau(1-r)} d r,
$$

whereupon resolvent (48) admits a unique solution $(q, u) \in \mathscr{D}(\mathscr{A})$ once we show the existence of a unique $q \in H^{2}(0, \ell)$ satisfying

$$
\left\{\begin{array}{l}
\alpha q_{x x}(x)-\beta q_{x}(x)-\lambda q(x)=-f(x)-\kappa \bowtie b(x), \\
q(0)=q_{x}(\ell)=0 .
\end{array}\right.
$$

Since $\lambda>0$ and recalling (35), one can readily check that the general solution of (51) is given by

$$
\begin{aligned}
q(x)= & l_{1} e^{\sigma_{1} x}+l_{2} e^{\sigma_{2} x}+\frac{1}{\sigma_{2}-\sigma_{1}} \int_{0}^{x}\left(e^{\sigma_{1}(x-s)}-e^{\sigma_{2}(x-s)}\right) \\
& \cdot(f(s)+\kappa \bowtie b(s)) d s .
\end{aligned}
$$

In (52), $l_{1}$ and $l_{2}$ are two constants that satisfy, thanks to the boundary conditions in (51), the following linear system:

$$
\left\{\begin{array}{l}
l_{1}+l_{2}=0 \\
\sigma_{1} e^{\sigma_{1} \ell} l_{1}+\sigma_{2} e^{\sigma_{2} \ell} l_{2}=M
\end{array}\right.
$$

in which

$$
M=\frac{1}{\sigma_{1}-\sigma_{2}} \int_{0}^{\ell}\left(\sigma_{1} e^{\sigma_{1}(\ell-s)}-\sigma_{2} e^{\sigma_{2}(\ell-s)}\right)(f(s)+\kappa \bowtie b(s)) d s .
$$

We suppose that $\lambda>0$ is large enough so that $\sigma_{2} e^{\sigma_{2} \ell}-\sigma_{1} e^{\sigma_{1} \ell} \neq 0$. Accordingly, the above linear system has a unique solution:

$$
l_{1}=-\frac{M}{\sigma_{2} e^{\sigma_{2} \ell}-\sigma_{1} e^{\sigma_{1} \ell}}, \quad l_{2}=\frac{M}{\sigma_{2} e^{\sigma_{2} \ell}-\sigma_{1} e^{\sigma_{1} \ell}} .
$$

Combining the last findings with (52) yields

$$
\begin{aligned}
q(x)= & \frac{1}{\sigma_{2}-\sigma_{1}} \int_{0}^{x}\left[e^{\sigma_{1}(x-s)}-e^{\sigma_{2}(x-s)}\right][f(s)+\kappa \bowtie b(s)] d s \\
& -\frac{M\left(e^{\sigma_{1} x}-e^{\sigma_{2} x}\right)}{\sigma_{2} e^{\sigma_{2} \ell}-\sigma_{1} e^{\sigma_{1} \ell}} .
\end{aligned}
$$

This clearly gives

$$
\begin{aligned}
q(\ell)= & \frac{1}{\sigma_{2}-\sigma_{1}} \int_{0}^{\ell}\left[e^{\sigma_{1}(\ell-s)}-e^{\sigma_{2}(\ell-s)}\right][f(s)+\kappa \bowtie b(s)] d s \\
& -\frac{M\left(e^{\sigma_{1} \ell}-e^{\sigma_{2} \ell}\right)}{\sigma_{2} e^{\sigma_{2} \ell}-\sigma_{1} e^{\sigma_{1} \ell}},
\end{aligned}
$$

which together with (50) implies that

$$
\begin{aligned}
q(\ell)\left[1+\frac{\kappa e^{-\lambda \tau} e^{\left(\sigma_{1}+\sigma_{2}\right) \ell}}{\left(\sigma_{2} e^{\sigma_{2} \ell}-\sigma_{1} e^{\sigma_{1} \ell}\right)} \int_{0}^{\ell}\left(e^{-\sigma_{2} s}-e^{-\sigma_{1} s}\right) b(s) d s\right] \\
=\frac{e^{\left(\sigma_{1}+\sigma_{2}\right) \ell}}{\left(\sigma_{2} e^{\sigma_{2} \ell}-\sigma_{1} e^{\sigma_{1} \ell}\right)} \int_{0}^{\ell}\left(e^{-\sigma_{1} s}-e^{-\sigma_{2} s}\right) \\
\cdot\left(f(s)+\kappa \tau b(s) \int_{0}^{1} g(r) e^{-\lambda \tau(1-r)} d r\right) d s .
\end{aligned}
$$

In light of (35), the latter becomes

$$
\begin{aligned}
& q(\ell)\left[1+\frac{2 \alpha \kappa e^{(\beta \ell / \alpha)-\lambda \tau}}{\left(\beta+\sqrt{G_{\lambda}}\right) e^{\left(\left(\beta+\sqrt{G_{\lambda}}\right) /(2 \alpha)\right) \ell}+\left(\sqrt{G_{\lambda}}-\beta\right) e^{\left(\left(\beta-\sqrt{G_{\lambda}}\right) /(2 \alpha)\right) \ell}} \int_{0}^{\ell}\left(e^{-\left(\left(\beta+\sqrt{G_{\lambda}}\right) /(2 \alpha)\right) s}-e^{\left(\left(\sqrt{G_{\lambda}}-\beta\right) /(2 \alpha)\right) e s}\right) b(s) d s\right] \\
&=\frac{2 \alpha e^{\beta \ell / \alpha}}{\left(\beta+\sqrt{G_{\lambda}}\right) e^{\left(\left(\beta+\sqrt{G_{\lambda}}\right) /(2 \alpha)\right) \ell}+\left(\sqrt{G_{\lambda}}-\beta\right) e^{\left(\left(\beta-\sqrt{G_{\lambda}}\right) /(2 \alpha)\right) e} \times \int_{0}^{\ell}\left(e^{\left(\left(\sqrt{G_{\lambda}}-\beta\right) /(2 \alpha)\right) e s}-e^{-\left(\left(\beta+\sqrt{G_{\lambda}}\right) /(2 \alpha)\right) s}\right)} \\
& \cdot\left(f(s)+\kappa \tau b(s) \int_{0}^{1} g(r) e^{-\lambda \tau(1-r)} d r\right) d s .
\end{aligned}
$$

Consequently, $q(\ell)$ is uniquely determined if and only if the following expression

$$
\begin{gathered}
1+\frac{2 \alpha \kappa e^{(\beta \ell / \alpha)-\lambda \tau}}{\left(\beta+\sqrt{G_{\lambda}}\right) e^{\left(\left(\beta+\sqrt{G_{\lambda}}\right) /(2 \alpha)\right) \ell}+\left(\sqrt{G_{\lambda}}-\beta\right) e^{\left(\left(\beta-\sqrt{G_{\lambda}}\right) /(2 \alpha)\right) \ell}} \\
\cdot \int_{0}^{\ell}\left(e^{-\left(\left(\beta+\sqrt{G_{\lambda}}\right) /(2 \alpha)\right) s}-e^{\left(\left(\sqrt{G_{\lambda}}-\beta\right) /(2 \alpha)\right) \ell s}\right) b(s) d s,
\end{gathered}
$$

is not zero, which leads us to claim that resolvent (48) has a unique solution $(q, u)$ given by (49) and (56). Whereupon, the operator $(\lambda I-\mathscr{A})^{-1}$ exists for $\lambda>0$ and $(\lambda I-\mathscr{A})^{-1} \in \mathscr{L}(\mathscr{H})$. Furthermore, by virtue of the Sobolev embedding theorem [40], the operator $(\lambda I-\mathscr{A})^{-1}$ is compact on $\mathscr{H}$, for $\lambda>0$. Finally, the spectrum of $\mathscr{A}$ consists of isolated eigenvalues with finite multiplicities and $(\lambda I-\mathscr{A})^{-1}$ is compact for any $\lambda \in \rho(\mathscr{A})$ [41]. 
We now turn to the eigenvalue problem. We have the following result.

\section{Theorem 6}

(i) $\lambda=0$ is an eigenvalue of the operator $\mathscr{A}$ if and only if the feedback gain obeys the following condition:

$$
\beta=\kappa \int_{0}^{\ell}\left(1-e^{-(\beta / \alpha) s}\right) b(s) d s .
$$

(ii) $\lambda=-\beta /\left(4 \alpha^{2}\right)$ is an eigenvalue of the operator $\mathscr{A}$ if and only if the feedback gain satisfies

$$
\kappa e^{\beta^{2} \tau /(4 \alpha)} \int_{0}^{\ell} s e^{-(\beta s /(2 \alpha))} b(s) d s=e^{-(\beta \ell /(2 \alpha))}\left(1+\frac{\beta \ell}{2 \alpha}\right) .
$$

(iii) If $\beta \neq \kappa \int_{0}^{\ell}\left(1-e^{-(\beta / \alpha) s}\right) b(s) d s$ and $1+(\beta / \alpha) \ell \neq$ $\kappa e^{(\beta / \alpha) l} \int_{0}^{\ell} s e^{-(\beta / \alpha) s} b(s) d s$, then the spectrum $\sigma(\mathscr{A})$ consists of eigenvalues with finite multiplicity and

$$
\sigma(\mathscr{A})=\{\lambda \in \mathbb{C} ; \Delta(\lambda)=0\},
$$

where

$$
\Delta(\lambda):=1+\frac{2 \alpha \kappa e^{((\beta e) / \alpha)-\lambda \tau}}{\left(\beta+\sqrt{G_{\lambda}}\right) e^{\left(\left(\beta+\sqrt{G_{\lambda}}\right) /(2 \alpha)\right) \ell}+\left(\sqrt{G_{\lambda}}-\beta\right) e^{\left(\left(\beta-\sqrt{G_{\lambda}}\right) /(2 \alpha)\right) \ell}} \int_{0}^{\ell}\left(e^{-\left(\left(\beta+\sqrt{G_{\lambda}}\right) /(2 \alpha)\right) s}-e^{\left(\left(\sqrt{G_{\lambda}}-\beta\right) /(2 \alpha)\right) e s}\right) b(s) d s,
$$

and $G_{\lambda}$ is defined by (35).

Proof. Let $\lambda \in \mathbb{C}$. The eigenvalue problem of $\mathscr{A}$ consists in seeking a nontrivial element $(q, u) \in \mathscr{D}(\mathscr{A})$ such that

$$
\left\{\begin{array}{l}
\alpha q_{x x}(x)-\beta q_{x}(x)-\lambda q(x)+\kappa b(x) u(1)=0 \\
u_{\rho}(\rho)+\lambda \tau u(\rho)=0 \\
u(0)=q(\ell), q(0)=q_{x}(\ell)=0 .
\end{array}\right.
$$

One can check from the second equation of (65) that $u$ is given by

$$
u(\rho)=q(\ell) e^{-\lambda \tau \rho} .
$$

Herewith, it suffices to find a nontrivial solution $q \in H^{2}(0, \ell)$ to the system

$$
\left\{\begin{array}{l}
\alpha q_{x x}(x)-\beta q_{x}(x)-\lambda q(x)+\kappa b(x) q(\ell) e^{-\lambda \tau}=0, \\
q(0)=q_{x}(\ell)=0 .
\end{array}\right.
$$

We shall treat 3 cases.

Case 1. A straightforward computation shows that $\lambda=0$ is an eigenvalue if and only if

$$
\begin{aligned}
q(x)= & \frac{\kappa}{\beta} q(\ell)\left(e^{\beta x / \alpha}-1\right) \int_{0}^{\ell} e^{-\beta s / \alpha} b(s) d s+\frac{\kappa}{\beta} q(\ell) \\
& \cdot \int_{0}^{x}\left(e^{(\beta / \alpha)(x-s)}-1\right) b(s) d s .
\end{aligned}
$$

Consequently,

$$
q(\ell)\left[1-\frac{\kappa}{\beta} \int_{0}^{\ell}\left(1-e^{-\beta s / \alpha}\right) b(s) d s\right]=0 .
$$

Thereby, we conclude that $\lambda=0$ is an eigenvalue of the system if and only if the feedback gain satisfies the following condition:

$$
\kappa=\beta\left(\int_{0}^{\ell}\left(1-e^{-\beta s / \alpha}\right) b(s) d s\right)^{-1} .
$$

Case 2. It is not difficult to verify from (67) that $\lambda=-\beta^{2} /(4 \alpha)$ is an eigenvalue if and only if

$$
\begin{aligned}
q(x)= & -\frac{\kappa \beta^{\beta^{2} \tau /(4 \alpha)}}{2 \alpha+\beta \ell} q(\ell)\left(\int_{0}^{\ell}[2 \alpha+\beta(\ell+s)] e^{-(\beta s /(2 \alpha))} b(s) d s\right) x e^{\beta x /(2 \alpha)} \\
& +\kappa q(\ell) e^{\beta^{2} \tau /(4 \alpha)} e^{\beta x /(2 \alpha)}\left(\int_{0}^{x}(s-x) e^{-\beta s /(2 \alpha)} b(s) d s\right) .
\end{aligned}
$$

Accordingly, $\lambda=-\beta /\left(4 \alpha^{2}\right)$ is an eigenvalue if and only if the feedback gain verifies

$$
\kappa e^{\left(\beta^{2} \tau /(4 \alpha)\right)} \int_{0}^{\ell} s e^{-(\beta s /(2 \alpha))} b(s) d s=e^{-(\beta \ell /(2 \alpha))}\left(1+\frac{\beta \ell}{2 \alpha}\right) .
$$

The assertion (ii) is then proved.

Case 3. Assume that $\lambda \notin\left\{0,-\left(\beta /\left(4 \alpha^{2}\right)\right)\right\}$. Thereby, (67) gives

$$
\begin{aligned}
q(x)= & a_{1} e^{\sigma_{1} x}+a_{2} e^{\sigma_{2} x}+\frac{\alpha}{\sqrt{G_{\lambda}}} \kappa q(\ell) e^{-\lambda \tau} \\
& \cdot \int_{0}^{x}\left(e^{\sigma_{1}(x-s)}-e^{\sigma_{2}(x-s)}\right) b(s) d s,
\end{aligned}
$$

where $G_{\lambda}, \sigma_{1}$, and $\sigma_{2}$ are given by (35). Moreover, $a_{1}$ and $a_{2}$ are constants to be determined by the boundary conditions $q(0)=q_{x}(\ell)=0$. In fact, we have

$$
\left\{\begin{array}{l}
a_{1}+a_{2}=0, \\
\sigma_{1} e^{\sigma_{1} \ell} a_{1}+\sigma_{2} e^{\sigma_{2} \ell} a_{2}=\Upsilon,
\end{array}\right.
$$


with

$$
\Upsilon=\frac{\alpha}{\sqrt{G_{\lambda}}} \kappa q(\ell) e^{-\lambda \tau} \int_{0}^{\ell}\left(\sigma_{1} e^{\sigma_{1}(\ell-s)}-\sigma_{2} e^{\sigma_{2}(\ell-s)}\right) b(s) d s .
$$

If $\sigma_{1} e^{\sigma_{1} \ell}=\sigma_{2} e^{\sigma_{2} \ell}$, then necessarily $\int_{0}^{\ell}\left(e^{-\sigma_{1} s}-e^{-\sigma_{2} s}\right) b(s) d s=0$. However, if $\sigma_{1} e^{\sigma_{1} \ell} \neq \sigma_{2} e^{\sigma_{2} \ell}$, then the solution $q(x)$ obtained in (73) takes the following form:

$$
\begin{aligned}
q(x)= & \frac{1}{\sigma_{2}-\sigma_{1}} \kappa q(\ell) e^{-\lambda \tau} \int_{0}^{x}\left[e^{\sigma_{1}(x-s)}-e^{\sigma_{2}(x-s)}\right] b(s) d s \\
& +\frac{\Upsilon\left(e^{\sigma_{1} x}-e^{\sigma_{2} x}\right)}{\sigma_{2} e^{\sigma_{2} \ell}-\sigma_{1} e^{\sigma_{1} \ell}} .
\end{aligned}
$$

Substituting $\ell$ for $x$ in (76) and recalling (35), we finally obtain that $\lambda$ obeys the following condition:

$$
1+\frac{2 \alpha \kappa e^{(\beta \ell / \alpha)-\lambda \tau} \int_{0}^{\ell}\left(e^{-\left(\left(\beta+\sqrt{G_{\lambda}}\right) /(2 \alpha)\right) s}-e^{\left(\left(\sqrt{G_{\lambda}}-\beta\right) /(2 \alpha)\right) \ell s}\right) b(s) d s}{\left(\beta+\sqrt{G_{\lambda}}\right) e^{\left(\left(\beta+\sqrt{G_{\lambda}}\right) /(2 \alpha)\right) \ell}+\left(\sqrt{G_{\lambda}}-\beta\right) e^{\left(\left(\beta-\sqrt{G_{\lambda}}\right) /(2 \alpha)\right) \ell}}=0 .
$$

The third assertion is then proved.

\section{Stability of the Closed-Loop System}

The aim of this section is to investigate the stability of the closed-loop system (14) according to the values of $\kappa$. To proceed, we assume throughout this section that $\lambda \neq 0$ is not an eigenvalue of our system, that is, $\beta \neq \kappa \int_{0}^{\ell}\left(1-e^{-\beta / \alpha s}\right) b(s) d s$ (see Theorem 6). Otherwise, the system is unstable by Theorem 3 .

Our first stability result is as follows.

Theorem 7. Suppose that the feedback gain $\kappa$ fulfills the condition

$$
|\kappa|<\frac{\pi}{\|b\| \ell} \sqrt{\frac{\alpha \beta}{2}} .
$$

Then, the $C_{0}$-semigroup $S(t)$ generated by the operator $\mathscr{A}$, defined by (21) and (22), is exponentially stable in $\mathscr{H}$. In other words, given any solution $q(x, t)$ of system (14), the following energy-norm

$$
\begin{aligned}
E_{1}(t):= & \frac{1}{2}\|(q(x, t), q(\ell, t-\tau \rho))\|_{\mathscr{H}}^{2}=\frac{1}{2}\left\{\int_{0}^{1} q^{2}(x, t) d x\right. \\
& \left.+\tau \beta \int_{0}^{1} q^{2}(\ell, t-\tau \rho) d \rho\right\},
\end{aligned}
$$

exponentially tends to zero as long as condition (78) is fulfilled.

Proof. Let $(q(x, t), u(\rho, t))$ be the unique solution of system (18) stemmed from an initial condition $\left(q_{0}, u_{0}\right)$ in $\mathscr{D}(\mathscr{A})$. Subsequently, differentiating (79) with respect to $t$, integrating by parts, and using the boundary conditions in (18), we get

$$
\begin{aligned}
E_{1}^{\prime}(t)= & -\alpha \int_{0}^{\ell} q_{x}^{2}(x, t) d x-\frac{\beta}{2} q^{2}(\ell, t-\tau) \\
& +\kappa q(\ell, t-\tau) \int_{0}^{\ell} q(x, t) b(x) d x .
\end{aligned}
$$

Next, evoking Young's and Cauchy-Schwartz inequalities yields (as for (43) and (44))

$$
E_{1}^{\prime}(t) \leq-\alpha \int_{0}^{\ell} q_{x}^{2}(x, t) d x+\frac{|\kappa|^{2}}{2 \beta}\|b\|^{2} \int_{0}^{\ell} q^{2}(x, t) d x .
$$

Furthermore, by virtue of Wirtinger's inequality (31), estimate (81) becomes

$$
E_{1}^{\prime}(t) \leq \frac{2|\kappa|^{2}\|b\|^{2} \ell^{2}-\alpha \beta \pi^{2}}{4 \beta \ell^{2}} \int_{0}^{\ell} q^{2}(x, t) d x .
$$

Thanks to assumption (78), one can write (82) as follows:

$$
E_{1}^{\prime}(t) \leq-\omega_{1} \int_{0}^{\ell} q^{2}(x, t) d x
$$

in which

$$
\omega_{1}=\frac{\alpha \beta \pi^{2}-2|\kappa|^{2}\|b\|^{2} \ell^{2}}{4 \beta \ell^{2}}>0 .
$$

On the other hand, let us introduce a new functional as

$$
E_{2}(t)=\varsigma \tau \int_{0}^{1} e^{-2 \gamma \tau \rho} q^{2}(\ell, t-\tau \rho) d \rho,
$$

where $\varsigma$ is a positive constant to be determined, while $\gamma$ is an arbitrary positive constant. One can check that

$$
\varsigma \tau \int_{0}^{1} e^{-2 \gamma \tau} q^{2}(\ell, t-\tau \rho) d \rho \leq E_{2}(t) \leq \varsigma \tau \int_{0}^{1} q^{2}(\ell, t-\tau \rho) d \rho .
$$

Moreover, differentiating (85), we have

$$
\begin{aligned}
E_{2}^{\prime}(t)= & 2 \varsigma \tau \int_{0}^{1} e^{-2 \gamma \tau \rho} q(\ell, t-\tau \rho) q_{t}(\ell, t-\tau \rho) d \rho \\
= & 2 \varsigma \tau \int_{0}^{1} e^{-2 \gamma \tau \rho} q(\ell, t-\tau \rho)\left(\frac{1}{\tau} q_{\rho}(\ell, t-\tau \rho)\right) d \rho \\
= & -\varsigma e^{-2 \gamma \tau} q^{2}(\ell, t-\tau)+\varsigma q^{2}(\ell, t) \\
& -2 \varsigma \tau \gamma \int_{0}^{1} e^{-2 \gamma \tau \rho} q^{2}(\ell, t-\tau \rho) d \rho .
\end{aligned}
$$


In view of the well-known inequality

$$
q^{2}(\ell, t) \leq \ell \int_{0}^{\ell} q_{x}^{2}(x, t) d x
$$

one can deduce from (87) that

$$
\begin{aligned}
E_{2}^{\prime}(t) \leq & -\varsigma e^{-2 \gamma \tau} q^{2}(\ell, t-\tau)+\varsigma \ell \int_{0}^{\ell} q_{x}^{2}(x, t) d x \\
& -2 \varsigma \tau \gamma \int_{0}^{1} e^{-2 \gamma \tau \rho} q^{2}(\ell, t-\tau \rho) d \rho .
\end{aligned}
$$

Now, let

$$
\mathscr{E}(t)=E_{1}(t)+E_{2}(t) .
$$

Using (79), (85), and (86), an elementary computation shows that there exist positive constants $L_{1}$ and $L_{2}$ such that for any $t \geq 0$, we have

$$
L_{1} E_{1}(t) \leq \mathscr{E}(t) \leq L_{2} E_{1}(t)
$$

Furthermore, differentiating (90) and using (83) and (89), we get

$$
\begin{aligned}
\mathscr{E}^{\prime}(t) \leq & -\varsigma e^{-2 \gamma \tau} q^{2}(\ell, t-\tau)-2 \varsigma \tau \gamma \int_{0}^{1} e^{-2 \gamma \tau \rho} q^{2}(\ell, t-\tau \rho) d \rho \\
& +\left(\varsigma \ell-\omega_{1}\right) \int_{0}^{\ell} q_{x}^{2}(x, t) d x,
\end{aligned}
$$

where $\omega_{1}$ is defined by (84). In view of (78), one can choose $\varsigma<\omega_{1} / \ell$ and hence (92) yields

$$
\mathscr{E}^{\prime}(t) \leq-\theta E_{1}(t)
$$

for some positive constant $\theta$ depending solely on the physical parameters $\alpha, \beta$, and $\ell$. Amalgamating (90), (91), and (93), we reach our desired result.

Our second stability result is as follows.

Theorem 8. Assume that the feedback gain $\kappa$ satisfies the condition

$$
|\kappa|<\frac{\pi \alpha}{2\|b\| \ell^{3 / 2} \sqrt{2 c_{1}+c_{2}}}
$$

for any constant $c_{1}>0$ and $c_{2}>1$. Then, the closed-loop system (23) is exponentially stable.

Proof. We begin by introducing the following functional:

$$
\begin{aligned}
\mathscr{F}(t)= & \frac{1}{2} \int_{0}^{1} q^{2}(x, t) d x+\frac{\tau \xi}{2} \int_{0}^{1} q^{2}(\ell, t-\tau \rho) d \rho \\
& +\nu \tau \int_{0}^{1} e^{-2 \gamma \tau \rho} q^{2}(\ell, t-\tau \rho) d \rho,
\end{aligned}
$$

where $\xi$ and $\nu$ are positive constants to be obtained and $\gamma$ is an arbitrary positive constant. Arguing as before, we have

$$
\begin{aligned}
\mathscr{F}^{\prime}(t) \leq & -\alpha \int_{0}^{\ell} q_{x}^{2}(x, t) d x+\left(\frac{1}{2}(\xi-\beta)+\nu\right) q^{2}(\ell, t) \\
& +\frac{|\kappa|}{2 K}\|b\|^{2} \int_{0}^{\ell} q^{2}(x, t) d x+\left[\frac{1}{2}(|\kappa| K-\xi)-v e^{-2 \gamma \tau}\right] \\
& \cdot q^{2}(\ell, t-\tau)-2 \nu \tau \gamma \int_{0}^{1} e^{-2 \gamma \tau \rho} q^{2}(\ell, t-\tau \rho) d \rho
\end{aligned}
$$

where $K$ is a positive constant related to the application of Young's inequality. Next, we invoke (31) and (88) and choose $\nu=c_{1}|\kappa| K$ and $\xi=c_{2}|\kappa| K$, where $c_{1}>0$ and $c_{2}>1$. Thereafter, (96) yields

$$
\begin{aligned}
\mathscr{F}^{\prime}(t)= & \frac{\left(2 c_{1}+c_{2}\right)|\kappa| \ell \pi^{2} K^{2}-2 \pi^{2} \alpha K+4|\kappa||| b||^{2} \ell^{2}}{2 \pi^{2} K} \\
& \cdot \int_{0}^{\ell} q_{x}^{2}(x, t) d x-\frac{\beta}{2} q^{2}(\ell, t)+\left[\frac{|\kappa|}{2} K\left(1-c_{2}\right)\right. \\
& \left.-v e^{-2 \gamma \tau}\right] q^{2}(\ell, t-\tau)-2 v \tau \gamma \int_{0}^{1} e^{-2 \gamma \tau \rho} q^{2}(\ell, t-\tau \rho) d \rho .
\end{aligned}
$$

In the light of (94), one can choose the positive constant $K$ such that

$$
K \in\left(\frac{\pi \alpha-\sqrt{\pi^{2} \alpha^{2}-4\left(2 c_{1}+c_{2}\right)|\kappa|^{2}\|b\|^{2} \ell^{3}}}{\pi\left(2 c_{1}+c_{2}\right)|\kappa| \ell}, \frac{\pi \alpha-\sqrt{\pi^{2} \alpha^{2}-4\left(2 c_{1}+c_{2}\right)|\kappa|^{2}\|b\|^{2} \ell^{3}}}{\pi\left(2 c_{1}+c_{2}\right)|\kappa| \ell}\right),
$$

so that the coefficient of $\int_{0}^{1} q_{x}^{2}(x, t) d x$ in (97) is negative. Thereby, (97) together with (31) implies that

$$
\mathscr{F}^{\prime}(t) \leq-\varrho E_{1}(t),
$$

where $E_{1}(t)=(1 / 2)\|(q(x, t), q(\ell, t-\tau \rho))\|_{\mathscr{H}}^{2}($ see $(79))$ and $\varrho$ is a negative constant depending solely on the physical parameters $\alpha, \beta$, and $\ell$. Finally, it is easy to check that $\mathscr{F}(t)$ satisfies similar inequalities to (91) and hence the exponential stability of the system follows.

\section{Remark 2}

(a) We emphasize on the fact that if the value of the feedback gain $\kappa$ increases, then the decay in Theorems 7 and 8 is slower. This is as a direct consequence of (78) (see also (94)). In turn, such conditions often occur for systems with time delay (see $[27,28,33,34,44]$ ) and can be viewed as a "smallness" of the delay term. 


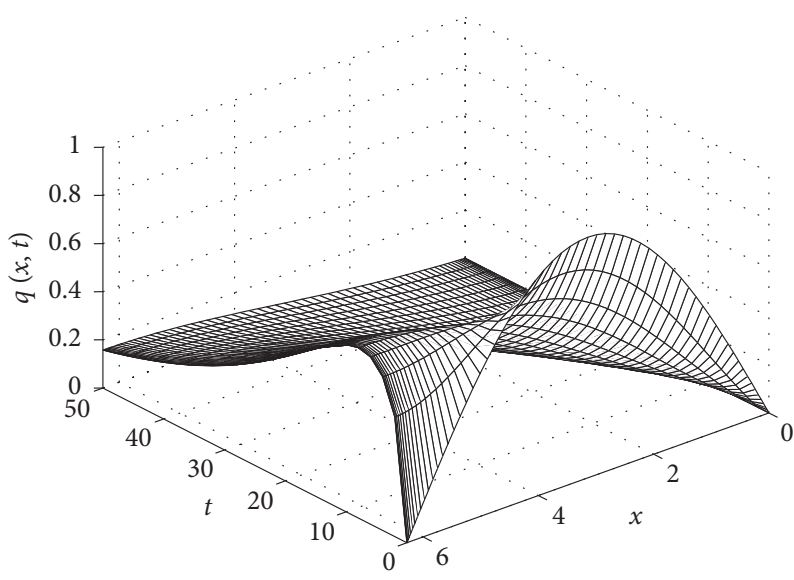

(a)

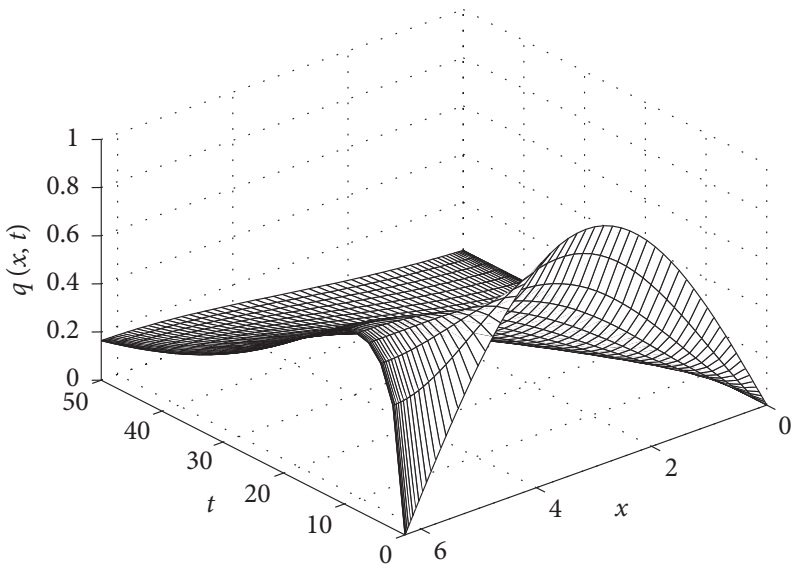

(c)

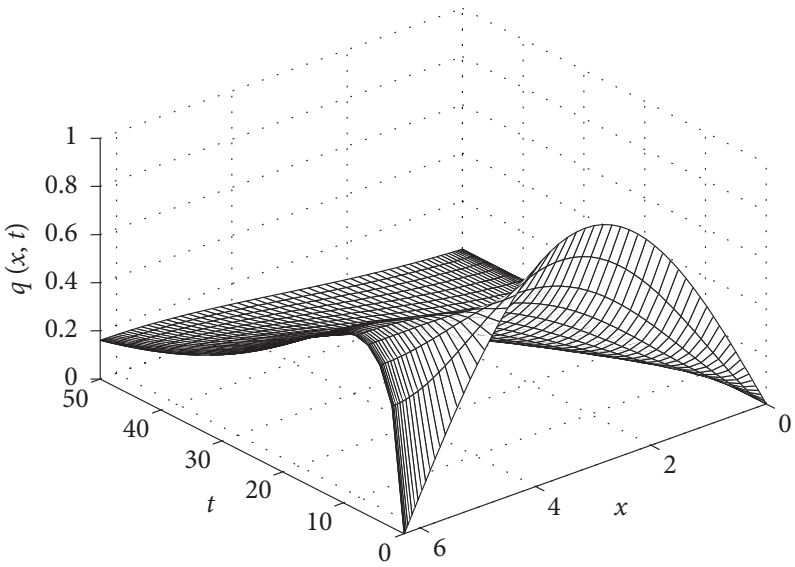

(b)

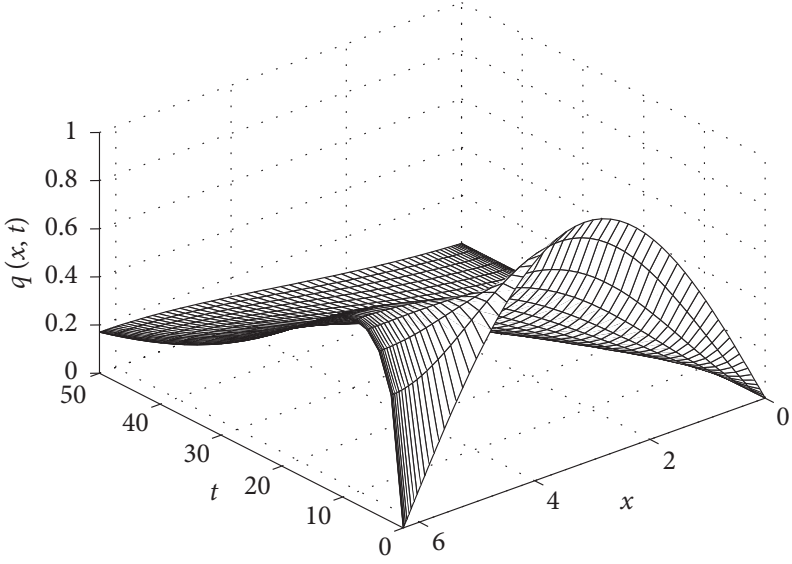

(d)

Figure 1: Time evolution of $q(x, t)$ in (14) with the spatial distribution function $b(x)=x / \pi-1, q(x, s)=(1+s) \sin (x / 2)$, $\alpha=0.4, \beta=0.1, \kappa=0.02$, and for different values of $\tau$ : (a) $\tau=0.02$; (b) $\tau=0.04$; (c) $\tau=0.06$; (d) $\tau=0.1$.

(b) It is also worth mentioning that the stability result obtained in Theorems 7 and 8 is valid without any restriction on the positive constant $\gamma$. Whence, $\gamma$ can be tuned in such a way the decay occurs as we desire.

\section{Numerical Results}

The discretization of the closed-loop system (14) is given by $\frac{\partial q^{N}}{\partial t}-\alpha \frac{\partial^{2} q^{N}}{\partial x^{2}}+\beta \frac{\partial q^{N}}{\partial x}+\left.\kappa q^{N}(\ell, t-\tau) b(x)\right|_{x=x_{j}}=0 ; j=1, \ldots, N-1$,

with

$$
\begin{aligned}
q^{N}(0, t) & =0, \frac{\partial q^{N}}{\partial x}(\ell, t)=0, t>0, \\
q^{N}\left(x_{j}, s\right) & =f\left(x_{j}, s\right), \quad j=0, \ldots, N, \quad-\tau \leq s \leq 0,
\end{aligned}
$$

in which $q^{N}$ is the discrete solution given by its value at the grid points $x_{j}$. Indeed, the expression of $q^{N}$ involves the Chebyshev series as follows:

$$
q^{N}(x, t)=\sum_{k=0}^{N} \widehat{q}_{k}(t) T_{k}(x)
$$

where for each $j=0, \ldots, N$, the function $T_{j}(x)$ is the Chebyshev polynomial of the first kind, which is defined as the eigenfunction of the singular Sturm-Liouville differential equation:

$$
\frac{d}{d x}\left(\sqrt{1-x^{2}} \frac{d T_{k}}{d x}(x)\right)+\frac{k^{2}}{\sqrt{1-x^{2}}} T_{k}(x)=0 .
$$

In the above equation,

$$
T_{k}(x)=\cos k \theta \text { and } \theta=\operatorname{arc}(\cos x) .
$$

The transformation $\tilde{x}=(2 x / \ell)-1$ will be applied on $(100)$ and $(102)$ so that the spatial domain $[0, \ell]$ becomes $[-1,1]$.

In the numerical simulations presented in this section, the Chebyshev collocation method (see [45] for more details) combined with backward Euler method and the Gauss-Lobatto points given by 


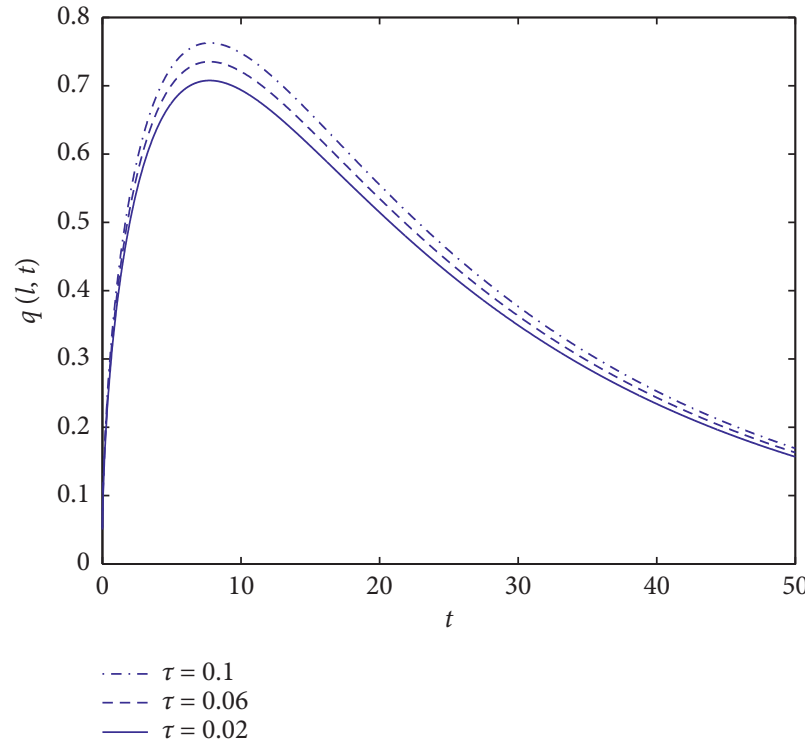

(a)

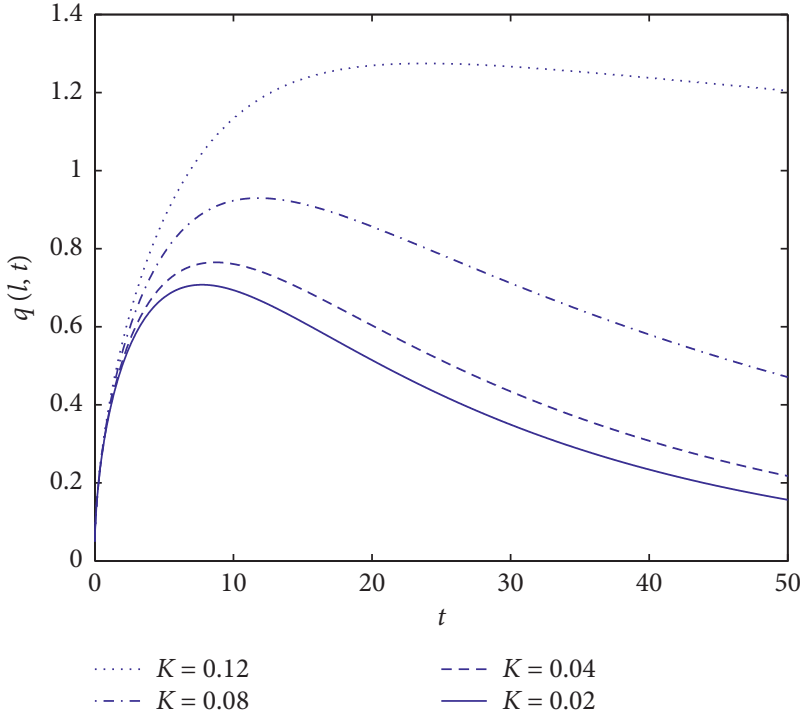

(b)

Figure 2: (a) $q(\ell, t)$ vs. time when $b(x)=(x / \pi)-1, q(x, s)=(1+s) \sin (x / 2), \alpha=0.4, \beta=0.1, \kappa=0.02$, and for different values of $\tau$. (b) $q(\ell, t)$ vs. time when $\alpha=0.4, \beta=0.1, \tau=0.02$, and for different values of $\kappa$.

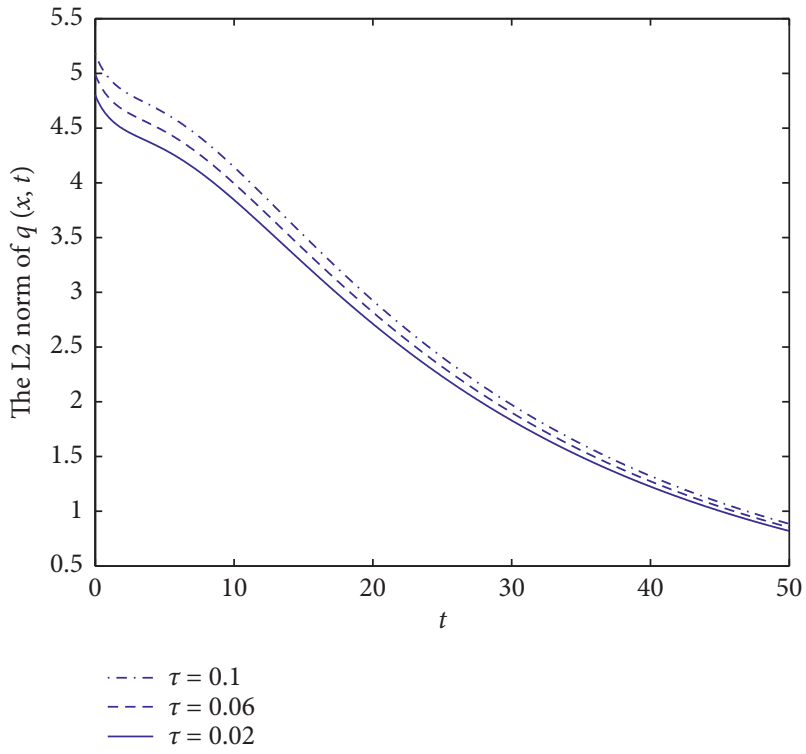

(a)



(b)

Figure 3: (a) The $L^{2}$-norm of $q(x, t)$ vs. time with $b(x)=x / \pi-1, q(x, s)=(1+s) \sin (x / 2), \alpha=0.4, \beta=0.1, \kappa=0.02$ and for different values of $\tau$. (b) The $L^{2}$-norm of $q(x, t)$ vs. time when $\alpha=0.4, \beta=0.1, \tau=0.02$, and for different values of $\kappa$.

$$
x_{j}=\cos \left(\frac{j \pi}{N}\right), \quad j=0, \ldots, N
$$

are used to deal with the feedback time delay models (100)-(102) for different values of the delay and the physical parameters of the canal.

Figure 1 describes the behavior of the solution $q(x, t)$ when the distribution function $b(x)=(x / \pi)-1$, the initial condition is $q(x, s)=(1+s) \sin (x / 2)$, and the control gain $\kappa=0.02, \alpha=0.4$, and $\beta=0.1$, for different values of $\tau$. The output $q(\ell, t)$ versus time, for different values of $\tau$, is shown in Figure 2(a) and also in Figure 2(b) but for different values of $\kappa$. Figure 3 displays the $L^{2}$-norm of $q(x, t)$ versus time for different values of $\tau$ (Figure 3(a)) and for different values of $\kappa$ (Figure $3(\mathrm{~b})$ ). Note that when $\kappa=0.02$ and as the value of $\tau$ increases from $\tau=0.02$ to $\tau=0.1$, the output $q(\ell, t)$ slowly converges to zero. Also, when $\tau=0.02$ and as the value of $\kappa$ is increased from $\kappa=$ 0.02 to $\kappa=0.12$, the solution slowly converges to the zero solution. This validates the analytical results presented in Section 5, where we have used smallness conditions (78) and (94) (see also Remark 2). 




(a)

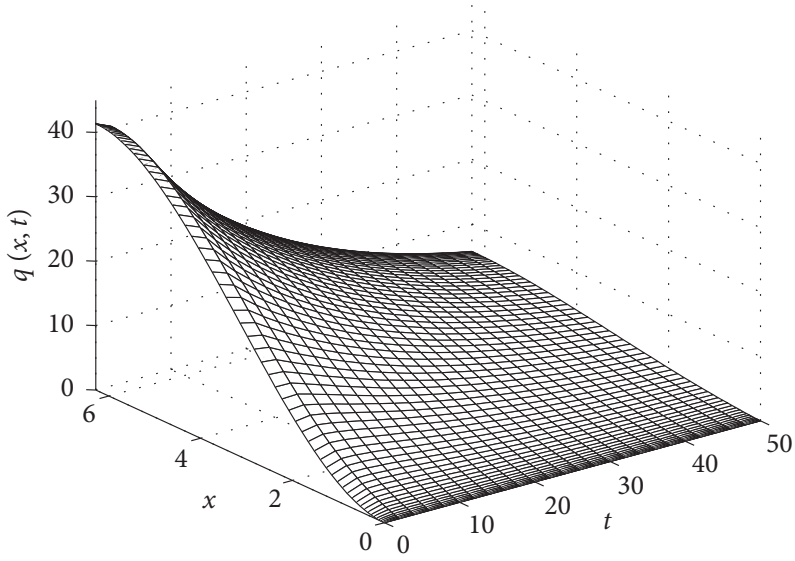

(c)

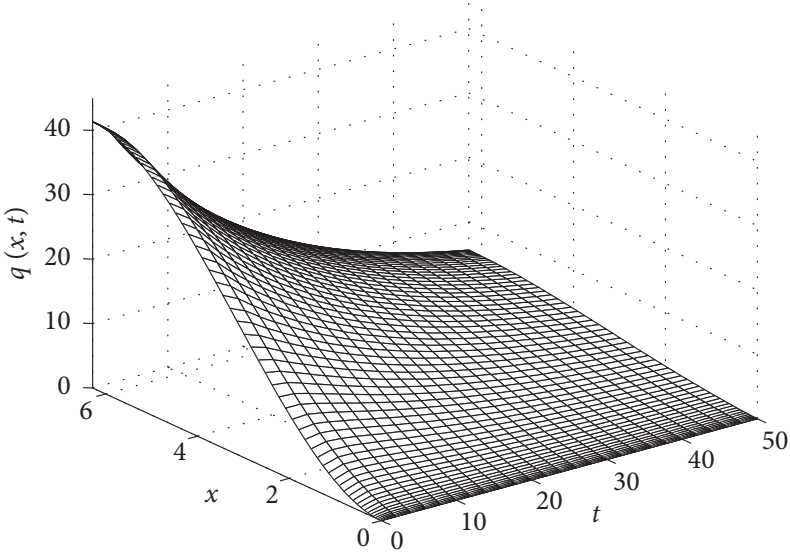

(b)

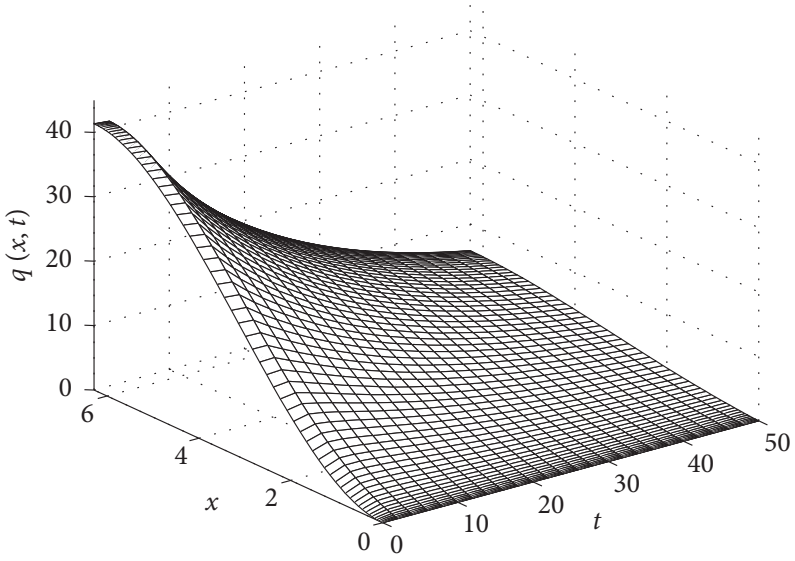

(d)

Figure 4: Time evolution of $q(x, t)$ in (14) when $b(x)=x / \pi-1, q(x, s)=(1+s)\left(\pi x^{2}-x^{3} / 3\right), \alpha=0.4, \beta=0.1, \kappa=0.02$, and for different values of $\tau$ : (a) $\tau=0.02$; (b) $\tau=0.04$; (c) $\tau=0.06$; (d) $\tau=0.1$.

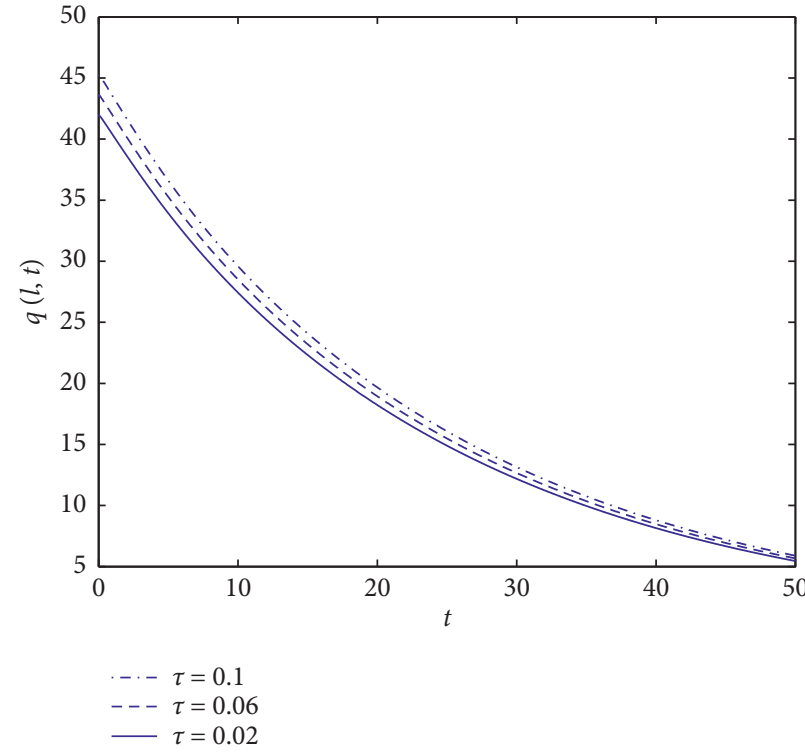

(a)



(b)

FIgURe 5: (a) $q(\ell, t)$ vs. time with the spatial distribution function $b(x)=(x / \pi)-1, q(x, s)=(1+s)\left(\pi x^{2}-x^{3} / 3\right), \alpha=0.4, \beta=0.1$, and $\kappa=$ 0.02 and for different values of $\tau$. (b) $q(\ell, t)$ vs. time when $\alpha=0.4, \beta=0.1, \tau=0.02$, and for different values of $\kappa$. 




(a)

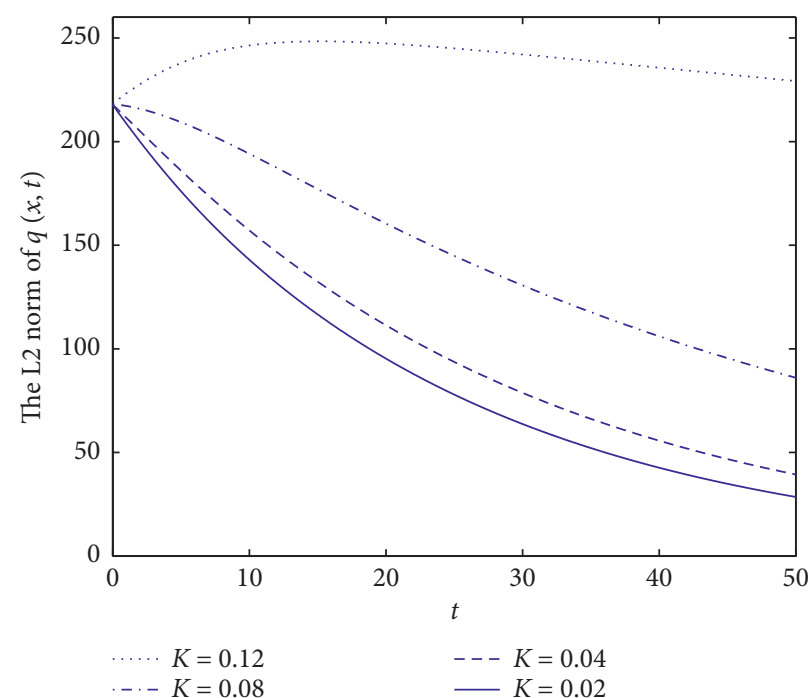

(b)

Figure 6: (a) The $L^{2}$-norm of $q(x, t)$ vs. time when $b(x)=(x / \pi)-1, q(x, s)=(1+s)\left(\pi x^{2}-x^{3} / 3\right), \alpha=0.4, \beta=0.1, \kappa=0.02$, and for different values of $\tau$. (b) The $L^{2}$-norm of $q(x, t)$ vs. time when $\alpha=0.4, \beta=0.1, \tau=0.02$, and for different values of $\kappa$.

Figures 4-6 are similar to Figures $1-3$ except that the initial conditions are $q(x, s)=(1+s)\left(\pi x^{2}-\left(x^{3} / 3\right)\right)$. Again the figures show that as the value of $\tau$ is increased, the output $q(\ell, t)$ slowly tends to zero, and this ties with our theoretical results.

\section{Conclusion}

In this article, an irrigation system has been considered when a constant time delay occurs in the flow control. Despite the presence of such a delay which could be a source of instability of the system or poor performance of the control, it has been shown that the impact of the delay can be eliminated provided the feedback gain satisfies some smallness conditions. Numerical results are also provided to demonstrate the correctness of the theoretical outcomes.

We would like to point out that it would be worthwhile to consider a time-dependent delay in the control and investigate the stability of the system. One may also investigate the stability of a networked system composed of $N$ channels (see $[46,47]$ for finite-dimensional systems). This will be the subject of a future work.

\section{Data Availability}

No data were used to support this study.

\section{Conflicts of Interest}

The authors declare that they have no conflicts of interest.

\section{References}

[1] B. de Saint-Venant, "Théorie du mouvement non permanent des eaux avec application aux crues des rivières et à l'introduction des marrées dans leur lit," Comptes rendus de l'Académie des Sciences, vol. 73, pp. 148-154, 1871.
[2] V. T. Chow, Open Channel Hydraulics, McGraw-Hill Book Company, New York, NY, USA, 1985.

[3] F. Benkhaldoun, S. Sari, and M. Seaid, "Projection finite volume method for shallow water flows," Mathematics and Computers in Simulation, vol. 118, pp. 87-101, 2015.

[4] X. Litrico and D. Georges, "Robust continuous-time and discrete-time flow control of a dam-river system. (I) Modelling," Applied Mathematical Modelling, vol. 23, no. 11, pp. 809-827, 1999.

[5] P. O. Malaterre, Modélisation, analyse et commande optimale $L Q R$ d'un canal d'irrigation, Ph.D. thesis, ENGREF, Paris, France, 1994.

[6] B. Chentouf and N. Smaoui, "Stability analysis and numerical simulations of a one dimensional open channel hydraulic system," Applied Mathematics and Computation, vol. 321, pp. 498-511, 2018.

[7] N. Jean-Baptiste, P.-O. Malaterre, C. Dorée, and J. Sau, "Data assimilation for real-time estimation of hydraulic states and unmeasured perturbations in a 1D hydrodynamic model," Mathematics and Computers in Simulation, vol. 81, no. 10, pp. 2201-2214, 2011.

[8] X. Litrico, V. Fromion, J.-P. Baume, C. Arranja, and M. Rijo, "Experimental validation of a methodology to control irrigation canals based on Saint-Venant equations," Control Engineering Practice, vol. 13, no. 11, pp. 1425-1437, 2005.

[9] X. Litrico and V. Fromion, "Analytical approximation of open-channel flow for controller design," Applied Mathematical Modelling, vol. 28, no. 7, pp. 677-695, 2004.

[10] X. Litrico and D. Georges, "Robust continuous-time and discrete-time flow control of a dam-river system. (II) Controller design," Applied Mathematical Modelling, vol. 23, no. 11, pp. 829-846, 1999.

[11] X. Litrico and V. Fromion, " $\mathrm{H}_{\infty}$ control of an irrigation canal pool with a mixed control politics," IEEE Transactions on Control Systems Technology, vol. 14, no. 1, pp. 99-111, 2006. 
[12] X. Litrico, "Robust IMC flow control of SIMO dam-river open-channel systems," IEEE Transactions on Control Systems Technology, vol. 10, no. 3, pp. 432-437, 2002.

[13] B. Cheviron and R. Moussa, "Determinants of modelling choices for 1-D free-surface flow and morphodynamics in hydrology and hydraulics: a review," Hydrology and Earth System Sciences, vol. 20, no. 9, pp. 3799-3830, 2016.

[14] C. Cholet, J.-B. Charlier, R. Moussa, M. Steinmann, and S. Denimal, "Assessing lateral flows and solute transport during floods in a conduit-flow-dominated karst system using the inverse problem for the advection-diffusion equation," Hydrology and Earth System Sciences, vol. 21, no. 7, pp. 3635-3653, 2017.

[15] P. Fan and J. C. Li, "Diffusive wave solutions for open channel flows with uniform and concentrated lateral inflow," $A d$ vances in Water Resources, vol. 29, no. 7, pp. 1000-1019, 2006.

[16] Y. B. Liu, S. Gebremeskel, F. De Smedt, L. Hoffmann, and L. Pfister, "A diffusive transport approach for flow routing in GIS-based flood modeling," Journal of Hydrology, vol. 283, no. 1-4, pp. 91-106, 2003.

[17] T. Moramarco, C. Pandolfo, and V. P. Singh, "Accuracy of kinematic wave and diffusion wave approximations for flood routing. I: steady analysis," Journal of Hydrologic Engineering, vol. 13, no. 11, pp. 1078-1088, 2008.

[18] R. Moussa and C. Bocquillon, "On the use of the diffusive wave for modelling extreme flood events with overbank flow in the floodplain," Journal of Hydrology, vol. 374, no. 1-2, pp. 116-135, 2009.

[19] C. W. Tsai, "Flood routing in mild-sloped rivers-wave characteristics and downstream backwater effect," Journal of Hydrology, vol. 308, no. 1-4, pp. 151-167, 2005.

[20] L. Wang, J. Q. Wu, W. J. Elliot, F. R. Fiedler, and S. Lapin, "Linear diffusion-wave channel routing using a discrete Hayami convolution method," Journal of Hydrology, vol. 509, pp. 282-294, 2014.

[21] G. Wu, E. W. M. Lee, and G. Li, "Numerical solutions of the reaction-diffusion equation," International Journal of $\mathrm{Nu}$ merical Methods for Heat \& Fluid Flow, vol. 25, no. 2, pp. 265-271, 2015.

[22] A. Bensoussan, G. D. Prato, M. Delfour, and S. K. Mitter, Representation and Control of Infinite-Dimensional Systems, Vol. I, Birkhäuser Boston, Inc., Boston, MA, USA, 1992.

[23] K. Ammari and B. Chentouf, "Further results on the robust regulation of a one-dimensional dam-river system," Journal of Optimization Theory and Applications, vol. 147, no. 3, pp. 597-606, 2010.

[24] B. Chentouf and J. M. Wang, "Stabilization of a one-dimensional dam-river system: nondissipative and noncollocated case," Journal of Optimization Theory and Applications, vol. 134, no. 2, pp. 223-239, 2007.

[25] B. Chentouf and J.-M. Wang, "A riesz basis methodology for proportional and integral output regulation of a one-dimensional diffusive-wave equation," SIAM Journal on Control and Optimization, vol. 47, no. 5, pp. 2275-2302, 2008.

[26] R. Datko, "Not all feedback stabilized hyperbolic systems are robust with respect to small time delays in their feedbacks," SIAM Journal on Control and Optimization, vol. 26, no. 3, pp. 697-713, 1988.

[27] R. Datko, "Two examples of ill-posedness with respect to time delays revisited," IEEE Transactions on Automatic Control, vol. 42, no. 4, pp. 511-515, 1997.

[28] R. Datko, J. Lagnese, and M. P. Polis, "An example on the effect of time delays in boundary feedback stabilization of wave equations," SIAM Journal on Control and Optimization, vol. 24, no. 1, pp. 152-156, 1986.

[29] F. Al-Musallam, K. Ammari, and B. Chentouf, "Asymptotic behavior of a $2 \mathrm{D}$ overhead crane with input delays in the boundary control," ZAMM-Journal of Applied Mathematics and Mechanics/Zeitschrift für Angewandte Mathematik und Mechanik, vol. 98, no. 7, pp. 1103-1122, 2018.

[30] K. Ammari and B. Chentouf, "Further results on the longtime behavior of a $2 \mathrm{D}$ overhead crane with a boundary delay: exponential convergence," Applied Mathematics and Computation, vol. 365, 2020.

[31] K. Ammari, S. Nicaise, and C. Pignotti, "Feedback boundary stabilization of wave equations with interior delay," Systems and Control Letters, vol. 59, no. 10, pp. 623-628, 2010.

[32] E. Fridman, S. Nicaise, and J. Valein, "Stabilization of second order evolution equations with unbounded feedback with time-dependent delay," SIAM Journal on Control and Optimization, vol. 48, no. 8, pp. 5028-5052, 2010.

[33] S. Nicaise and C. Pignotti, "Stability and instability results of the wave equation with a delay term in the boundary or internal feedbacks," SIAM Journal on Control and Optimization, vol. 45, no. 5, pp. 1561-1585, 2006.

[34] S. Nicaise and J. Valein, "Stabilization of second order evolution equations with unbounded feedback with delay," ESAIM: Control, Optimisation and Calculus of Variations, vol. 16, no. 2, pp. 420-456, 2010.

[35] G. Q. Xu, S. P. Yung, and L. K. Li, "Stabilization of wave systems with input delay in the boundary control," ESAIM: Control, Optimisation and Calculus of Variations, vol. 12, no. 4 , pp. 770-785, 2006.

[36] B. Chentouf, "Output feedback stabilization of a river irrigation canal," in Proceedings of the of the International Conference on Research in Mathematics, pp. 140-146, Malaysia, April 2007.

[37] C. M. Dafermos, "Asymptotic stability in viscoelasticity," Archive for Rational Mechanics and Analysis, vol. 37, no. 4, pp. 297-308, 1970.

[38] A. Pazy, Semigroups of Linear Operators and Applications to Partial Differential Equations, Springer-Verlag, New York, NY, USA, 1983.

[39] R. F. Curtain and H. J. Zwart, An Introduction to InfiniteDimensional Linear Systems Theory, Spring-Verlag, New York, NY, USA, 1995.

[40] R. Adams, Sobolev Spaces, Academic Press, 1975.

[41] T. Kato, Perturbation Theory of Linear Operators, SpringerVerlag, New York, NY, USA, 1976.

[42] F. L. Huang, "Characteristic conditions for exponential stability of linear dynamical system in Hilbert spaces," Annals of Differential Equations, vol. 1, pp. 43-53, 1985.

[43] G. H. Hardy, J. E. Littlewood, and G. Pólya, Inequalities, Cambridge University Press, Cambridge, UK, 2nd edition, 1988.

[44] B. Chentouf, "Stabilization of the rotating disk-beam system with a delay term in boundary feedback," Nonlinear Dynamics, vol. 78, no. 3, pp. 2249-2259, 2014.

[45] C. Canuto, M. Y. Hussaini, A. Quarteroni, and T. A. Zang, Spectral Methods in Fluid Dynamics, Springer-Verlag, Berlin, Germany, 1988.

[46] H. B. Liu and D. Q. Wang, "Stability and stabilisation of a class of networked dynamic systems," International Journal of Systems Science, vol. 49, no. 5, pp. 964-973, 2018.

[47] H. B. Liu and H. Yu, "Finite-time control of continuous-time networked dynamical systems," IEEE Transactions on Systems, Man, and Cybernetics: Systems, 2018. 